\title{
Zoo visitors affect sleep, displacement activities, and affiliative and aggressive behaviors in captive ebony langurs (Trachypithecus auratus)
}

\author{
Allison M. Roth ${ }^{1,2} \cdot$ Marina Cords ${ }^{1}$ \\ Received: 31 August 2019 / Revised: 20 February 2020 / Accepted: 27 February 2020 \\ (C) ISPA, CRL 2020
}

\begin{abstract}
Previous studies have shown that the number, noise level, and activity level of zoo visitors can negatively influence the behavior of captive animals. This study combined these three factors into a single visitor impact score and assessed whether visitor impact predicted the frequency or occurrence of displacement activities, affiliative behaviors, and aggression in a group of six captive ebony langurs (Trachypithecus auratus). This study also examined whether the amount of time the ebony langurs spent sleeping each day was correlated to the mean visitor impact score for that day. We used negative binomial and binomial models to analyze data collected during 5-min focal follows. Higher visitor impact scores predicted greater expression of displacement activities, affiliative behaviors, and aggression, suggesting that zoo visitors were a source autonomic arousal for the langurs. Similarly, the langurs spent more time sleeping on days with higher mean visitor impact scores, which may indicate learned helplessness. This study suggests that zoo visitors may be a source of environmental stress for captive ebony langurs. Nevertheless, the positive relationship between high visitor impact score and the occurrence of affiliative behavior types may indicate that the langurs use certain activities to decrease visitor-induced stress.
\end{abstract}

Keywords Visitor effects $\cdot$ Zoo biology $\cdot$ Primates $\cdot$ Animal welfare $\cdot$ Behavior

\section{Introduction}

Zoo visitors represent a vital source of revenue for zoos. Admission tickets and donations can further conservation, education, and research (Fernandez et al., 2009). Additionally, much of this revenue goes into maintaining exhibits and improving the welfare of the exhibited animals (Fernandez et al.,

Electronic supplementary material The online version of this article (https://doi.org/10.1007/s10211-020-00338-7) contains supplementary material, which is available to authorized users.

Allison M. Roth

amr2264@columbia.edu

Marina Cords

mc51@ columbia.edu

1 Department of Ecology, Evolution, and Environmental Biology, Columbia University, 10th Floor Schermerhorn Extension, MC 5557, 1200 Amsterdam Avenue, New York, NY 10027, USA

2 Department of Biology, University of Florida, 876 Newell Dr, Gainesville, FL 32611, USA
2009). A conflict may arise, however, if the presence of visitors has a negative impact on zoo animal welfare (Hosey, 2000). Many mammalian and avian taxa have been shown to exhibit behavioral/physiological changes in the presence of zoo visitors (Chamove et al., 1988; Davis et al., 2005; Larsen et al., 2014; Owen, 2004; Quadros et al., 2014; Sellinger and Ha, 2005; Sherwen et al., 2015). This phenomenon is known as the "visitor effect" (Hosey, 2000).

The presence of zoo visitors can be neutral or have enriching effects for some species (Cook and Hosey, 1995; Owen, 2004; Snyder, 1975). However, in most species for which a visitor effect has been observed, individuals exhibit physiological or behavioral changes that are interpreted as adverse in an animal welfare context (Chamove et al., 1988; Davis et al., 2005; Mallapur et al., 2005). For example, urinary cortisol levels of spider monkeys (Ateles geoffroyi rufiventris) and fecal cortisol levels of Indian blackbucks (Antilope cervicapra) may increase with increasing numbers of zoo visitors (Davis et al., 2005; Rajagopal et al., 2011). In other mammals, individuals display increased aggression (Chamove et al., 1988; Glatston et al., 1984; Mitchell et al., 1991), decreased affiliative behavior (Chamove et al., 1988; 
Glatston et al., 1984), and increased expression of abnormal behavior (Mallapur et al., 2005; Sellinger and Ha, 2005) with increasing number or density of visitors. Such behavioral changes may result from a heightened physiological stress response (Larsen et al., 2014; Mallapur et al., 2005).

The presence of zoo visitors can also influence the behavior of captive animals by increasing noise, distracting activity, and possibly through olfactory channels (Quadros et al., 2014). For example, brown howler monkeys (Alouatta guariba), golden-headed lion tamarins (Leontopithecus chrysomelas), chimpanzees (Pan troglodytes), and koalas (Phascolarctos cinereus) increase vigilance and/or movement when visitors are noisier (Larsen et al., 2014; Perret et al., 1995; Quadros et al., 2014). Similarly, some primates exhibit more audience-directed behaviors and/or increased movement when visitors are more active (Hosey and Druck, 1987; Mitchell et al., 1992; Perret et al., 1995).

In this study, we combined zoo visitor number, noise level, and activity level into a single visitor impact score and tested whether visitor impact predicted the frequency or occurrence of seven types of behavior in captive ebony langurs (Trachypithecus auratus). These behaviors included four displacement activities (scratching, body shaking, displacement yawning, and autogrooming; Maestripieri et al., 1992), two affiliative behaviors (mounting and allogrooming), and aggression. We hypothesized that zoo visitors would have a negative effect on ebony langur behavior. Displacement activities increase under stressful conditions (de Waal and Yoshihara, 1983; Hadidian, 1980; Troisi and Schino, 1987) and may indicate the emotional state of an individual (Kutsukake and Castles, 2001; Maestripieri et al., 1992; Troisi, 2002). Accordingly, we predicted that when visitor impact score was higher, the ebony langurs would be more likely to scratch, body shake, displacement yawn, autogroom, and aggress, and less likely to mount and allogroom.

We also tested whether mean daily visitor impact score would correspond to the amount of time the ebony langurs spent sleeping on a given day. Zoo animals may find it difficult to escape from potential sources of visitor-induced stress (e.g., noise, activity), resulting in increased sleep, rest, or other activities associated with learned helplessness (i.e., failure to cope with inescapable aversive stimuli; Wells, 2005, 2009; Wells et al., 2002). We predicted that the ebony langurs would spend more time sleeping on days with higher mean visitor impact scores.

\section{Methods}

\section{Study animals and exhibit}

Study subjects were ebony langurs (Trachypithecus auratus) kept in the JungleWorld building at the Bronx Zoo, New York
City (USA). The group included one male and five females, aged between 8 and 19 years old at the start of the study. The male was the father of all five females, and the females were either half or full siblings. We identified individuals by unique physical traits and randomly assigned each individual an identification number from one to six.

The langur exhibit was an indoor enclosure $\left(\sim 93 \mathrm{~m}^{2}\right)$ housing a variety of fauna (great Indian fruit bats (Pteropus giganteus), falcated ducks (Anas falcata), and Philippine ducks (Anas luzonica)) and flora (white mangroves (Laguncularia racemosa), which formed a network of elevated resting areas, torch ginger (Etlingera elatior), and other herbaceous and woody species) in addition to the langurs. Aside from the mangroves, the langurs used man-made boulders and outcrops to climb or rest. Visitors could view the monkeys from an 11.1-m-long corridor and a 1.2-m by 1.7$\mathrm{m}$ two-way window. Visitors at the window were easily visible from the corridor, and noise produced on the visitor side of the window was audible from the corridor. Alongside the corridor, a wet moat and 1.1-m-tall glass barrier separated zoo visitors and animals. An audio track of various rainforest noises (e.g., primate and avian vocalizations) played on a loop during public visiting hours.

\section{Data collection}

AMR conducted 612 5-min focal samples from the public viewing area during public visiting hours (10:00-17:00 h) on 19 days from September 23, 2014 to April 11, 2015. The mean \pm SD number of samples per individual was $102.0 \pm 0.9$ (range $=101-103)$, and the mean \pm SD number of samples per day was $32.2 \pm 9.9$ (range $=6-42$ ). Focal samples on the same individual were always separated by at least $1 \mathrm{~h}$, and the order in which individuals were sampled was randomized between days.

During each focal sample, AMR recorded all occurrences of four displacement activities (scratching, body shaking, displacement yawning, and autogrooming; as defined by Maestripieri et al. (1992)), two affiliative behaviors (mounting and allogrooming), and aggression (i.e., lunges, chases, and violent physical contact). Scratching occurred frequently throughout the focal samples, allowing a count of scratching events. Displacement yawning and aggression also occurred frequently enough to be recorded as count data. In contrast, body shaking, autogrooming, allogrooming, and mounting occurred less frequently, and we used binary records of these behaviors (yes/no) in our analyses. When a behavior involved more than one individual (i.e., mounting, allogrooming, and aggression), we included cases in which the focal individual either directed or received the behavior towards/from a conspecific. A focal individual that spent the entire 5-min observation period with its eyes closed was coded as sleeping. 
AMR also recorded the number, noise level, and activity level of zoo visitors during each focal sample. She recorded the total number of zoo visitors in the corridor and/or in front of the window during the focal sample by keeping track of visitors as they moved through the public viewing space. When a large school group resulted in a high rate of visitors entering the exhibit, she asked a chaperone to provide a head count. We scored noise level on an ordinal scale of zero to four with higher numbers indicating higher volume (Table 1), and we noted the highest level of noise produced by any single visitor during the span of each focal sample. We scored activity level on a similar ordinal scale of zero to four (Table 1), recording the highest level of activity of any single visitor, as for noise.

Several measures during data collection aimed to minimize the effects of observer presence on the langurs' behavior. (1) Before any data collection, AMR spent roughly $8 \mathrm{~h}$ spread over the course of 3 days sitting in view of the monkeys to habituate them to her presence. (2) She wore the same jacket and carried the same backpack on every visit to the exhibit to facilitate habituation. (3) Upon arriving at the exhibit each day, she spent ca. 10 min sitting in view of the langurs before recording data. (4) During the focal sampling, she tried to remain low to the ground and adopted a seated position whenever possible. Previous work has shown that several species of captive primates groom more, exhibit less agonistic behavior, and are more active when human observers are closer to the ground (Chamove et al., 1988).

\section{Data analysis}

We used R 3.5.2 (R Core Team 2018) for all analyses. We standardized the data for visitor number, noise level, and activity level and calculated the sum of the three standardized values to compute a visitor impact score for each focal sample. Because the visitor impact score included both auditory and visual cues, we removed samples where the focal individual was sleeping (i.e., its eyes were closed) when assessing the

Table 1 Ethogram for zoo visitor noise and activity levels
Noise level
0 Silent
1 Whispering
2 Talking at a normal conversational volume
3 Talking with a raised voice as if attempting to talk over something
4 Yelling, screaming, crying, banging noises

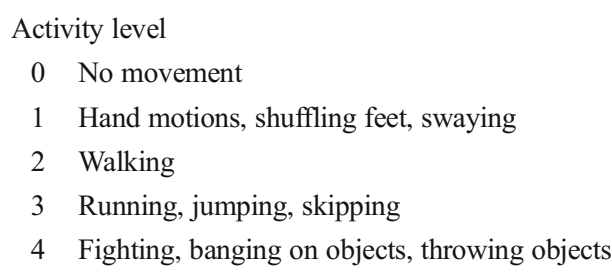

effects of visitor impact score on the various behavioral elements. For this set of analyses, we calculated visitor impact score using the reduced dataset (i.e., the dataset containing only samples where the focal individual was awake).

We ran negative binomial generalized linear mixed models to test for the effects of visitor impact on count data. Likelihood ratio tests favored the use of a negative binomial family over a Poisson family for these analyses (aggression: $\chi^{2}=34.484$, $p<0.001$; scratching: $\chi^{2}=20.680, p<0.001$; yawning: $\chi^{2}=$ $20.109, p<0.001)$. We ran separate models with aggression, scratching, and yawning (number of events per follow) as response variables. For each model, we included visitor impact score as a fixed effect and individual identity and date as random effects. Similarly, we ran generalized linear mixed models with a binomial family to test for the effects of visitor impact score on body shaking, autogrooming, allogrooming, and mounting. Here, each response variable was binary (yes/no), and we again included visitor impact score as a fixed effect and individual identity and date as random effects.

We also examined whether the proportion of focal samples in which the langurs spent sleeping each day could be predicted by the mean visitor impact score for that day. We ran a generalized linear model with a binomial family, using the daily ratio of focal samples spent sleeping versus focal samples spent awake as the response. On the first day of sampling, AMR conducted only six focal samples, and she waited until the ebony langurs were awake before beginning data collection. Because of this bias, we removed the first day of sampling from this analysis.

We used a single visitor impact score to avoid issues with multicollinearity. A Pearson's product-moment correlation demonstrated a strong positive correlation between visitor noise and activity level $(r=0.750 ; 95 \% \mathrm{CI}=0.0 .714-0.783$; $p<0.001$; $\mathrm{df}=610$ ), and moderate positive correlations between visitor number and noise level (noise $r=0.486 ; 95 \%$ $\mathrm{CI}=0.423-0.544 ; p<0.001 ; \mathrm{df}=610)$ and between visitor number and activity level $(r=0.400 ; 95 \% \mathrm{CI}=0.331-0.464$; $p<0.001$; $\mathrm{df}=610)$. Nevertheless, we acknowledge that it may be helpful from a management standpoint to understand the individual effects of visitor number, noise level, and activity level on captive ebony langur behavior. For this reason, we conducted three follow-up analyses that separately examined these effects. For these analyses, we ran models identical to the ones for the combined visitor impact score, but instead, we included visitor number, visitor noise, or visitor activity level as the fixed effect in place of visitor impact score.

\section{Results}

The langurs were awake for $77 \%$ of the focal samples and asleep for $23 \%$ of them $(N=612)$. When we excluded samples where the focal individual was sleeping, the mean \pm SD 
number of visitors was $11.9 \pm 15.0$ (range $0-79$ ), the mean \pm SD noise level was $2.3 \pm 1.4$ (range $0-4$ ), and the mean \pm SD activity level was $1.9 \pm 0.9$ (range $0-4$ ).

Each subject exhibited every behavior at least once during the study, but certain behavior types occurred more frequently than others. The langurs scratched themselves in $93 \%$ of the focal samples for which the animals were awake, and scratches occurred 0-23 times within a given focal sample (mean $\pm \mathrm{SD}=4.2 \pm 3.0$ ). The langurs exhibited displacement yawning in $7 \%$ of focal samples in which the subject was awake, with 0-8 yawns per focal sample (mean $\pm \mathrm{SD}=0.12$ \pm 0.56 ). Aggression was seen in $6 \%$ of focal samples in which the subject was awake, with 0-5 aggressions per focal sample (mean $\pm \mathrm{SD}=0.11 \pm 0.52$ ). Allogrooming occurred in $30 \%$ of focal samples in which the subject was awake, autogrooming in $27 \%$, body shaking in $15 \%$, and mounting in $4 \%$ of samples.

Visitor impact score had a significant effect on five of the seven behavior types (Table 2). Aggression, scratching, and displacement yawning were more frequent with larger visitor impact scores (Fig. 1, Table 2). Langurs were also more likely to allogroom and mount with higher visitor impact scores (Fig. 2, Table 2). Additionally, visitor impact score and body shaking were positively related, although this pattern was not quite significant (Fig. 2, Table 2). Visitor impact score had no significant effect on autogrooming (Fig. 2, Table 2). When we examined separate effects of visitor number, noise level, and activity level in follow-up analyses, results paralleled those found for models with a combined visitor impact score, except the positive relationship between visitor activity level and body shaking was now significant (Tables S1-S3). Different aspects of visitor behavior had stronger effects depending on the response variable in question. Aggression appeared to be most influenced by visitor number, scratching, allogrooming, and mounting appeared to be most affected by visitor noise, and body shaking appeared to be most influenced by visitor activity (Tables S1-S3).
The langurs also slept during a higher proportion of the focal samples on days with higher mean visitor impact scores (Est. $\pm \mathrm{SE}=0.184 \pm 0.056 ; 95 \% \mathrm{CI}=0.075-0.294 ; z$ value $=$ 3.307; $p=0.001$; Fig. 3). Relationships were similar when we examined the effects of mean visitor number (Est. $\pm \mathrm{SE}=$ $0.339 \pm 0.106 ; 95 \% \mathrm{CI}=0.129-0.546 ; z$ value $=3.194 ; p=$ 0.001 ), mean visitor noise level (Est. $\pm \mathrm{SE}=0.602 \pm 0.187$; $95 \% \mathrm{CI}=0.240-0.975 ; z$ value $=3.212 ; p=0.001)$, and mean visitor activity level (Est. $\pm \mathrm{SE}=0.607 \pm 0.220 ; 95 \% \mathrm{CI}=$ $0.188-1.051 ; z$ value $=2.763 ; p=0.006)$ on the proportion of focal samples spent sleeping, with mean visitor activity level having the greatest effect. However, given that there were 2 days with unusually high visitor impact scores (Fig. 3 ), we re-analyzed the data, omitting these points. Results held for mean visitor impact score (Est. $\pm \mathrm{SE}=0.221 \pm 0.097 ; 95 \%$ $\mathrm{CI}=0.035-0.416 ; z$ value $=2.279 ; p=0.023)$, mean visitor number (Est. $\pm \mathrm{SE}=0.882 \pm 0.305 ; 95 \% \mathrm{CI}=0.284-1.481 ; z$ value $=2.892 ; p=0.004)$, and mean visitor noise level (Est. \pm $\mathrm{SE}=0.508 \pm 0.244 ; 95 \% \mathrm{CI}=0.040-0.997 ; z$ value $=2.086$; $p=0.037$ ), but the relationship between mean visitor activity level and proportion of time spent sleeping was slightly nonsignificant (Est. $\pm \mathrm{SE}=0.446 \pm 0.243 ; 95 \% \mathrm{CI}=-0.018$ $0.936 ; z$ value $=1.836 ; p=0.066)$.

\section{Discussion}

Previous work has shown that the number, noise level, and activity level of zoo visitors can affect the behavior of zoo animals. This study tested whether the behavior of captive ebony langurs responds to a combination of these three factors. Consistent with the original predictions, the langurs scratched and yawned more when visitor impact was greater. Western lowland gorillas (Gorilla gorilla gorilla) also scratch more with higher numbers of visitors (Carder and Semple, 2008). Displacement activities are thought to be behavioral responses to stressful situations that indicate autonomic
Table 2 Effect of visitor impact score on the frequency of ebony langur aggressions, scratching, and displacement yawning, as well as the occurrence of langur body shaking, autogrooming, allogrooming, and mounting, in focal follows when the focal subject was awake

\begin{tabular}{llrrr}
\hline Behavior & Est. \pm SE & $z$ & $p$ & 95\% CI \\
\hline Count response variables & & & & \\
$\quad$ Aggression & $0.349 \pm 0.109$ & 3.191 & 0.001 & $0.109-0.564$ \\
Scratching & $0.181 \pm 0.013$ & 13.620 & $<0.001$ & $0.157-0.208$ \\
$\quad$ Displacement yawning & $0.254 \pm 0.080$ & 3.171 & 0.002 & $0.092-0.418$ \\
Binary response variables & & & & \\
Body shaking & $0.102 \pm 0.053$ & 1.930 & 0.054 & $-0.002-0.206$ \\
Autogrooming & $0.044 \pm 0.048$ & 0.910 & 0.363 & $-0.052-0.139$ \\
Allogrooming & $0.193 \pm 0.044$ & 4.361 & $<0.001$ & $0.107-0.288$ \\
Mounting & $0.466 \pm 0.118$ & 3.964 & $<0.001$ & $0.217-0.727$ \\
\hline
\end{tabular}

$p$ values for significant effects are italicized. See text for details $(N=472)$ 
a

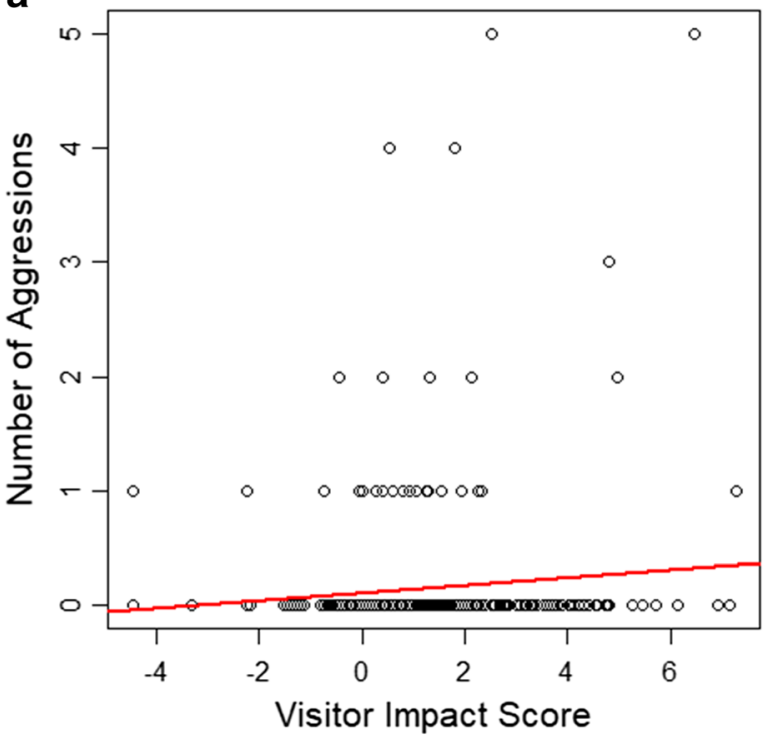

b

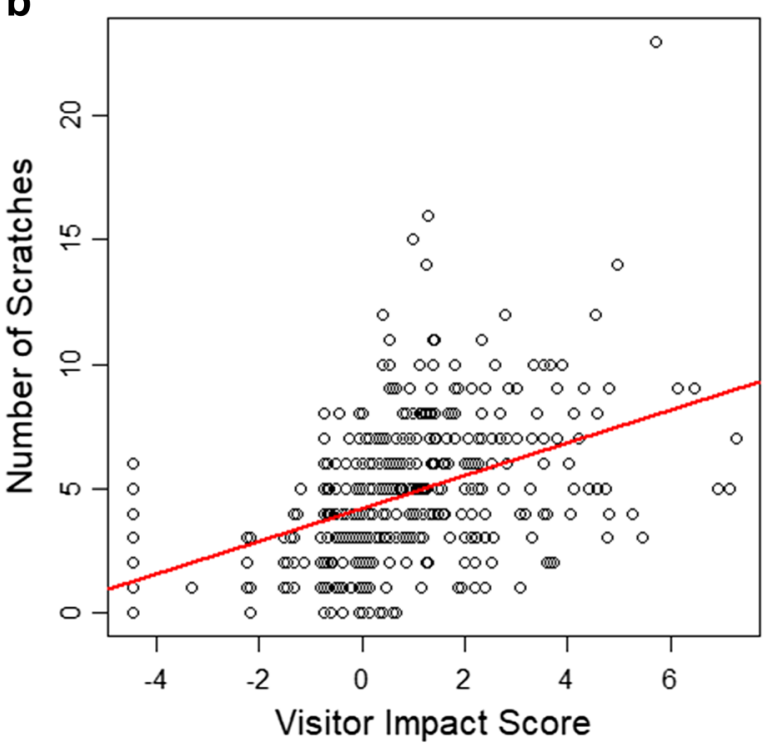

C

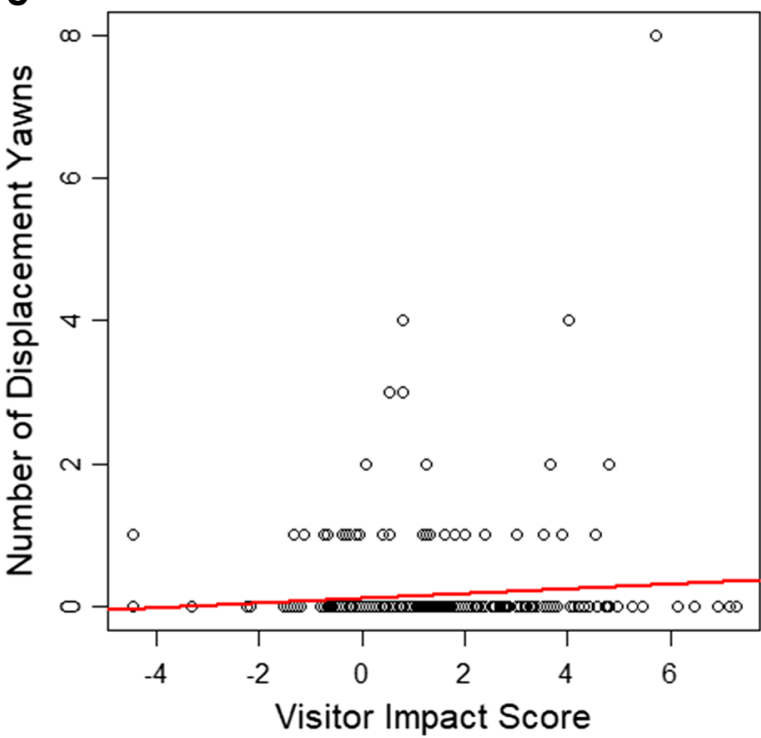

Fig. 1 Relationships between visitor impact score and the a number of aggressions given/received, $\mathbf{b}$ number of scratches, and $\mathbf{c}$ number of displacement yawns ebony langurs exhibited per 5-min focal sample. The regression lines predicted by the models are shown.

arousal (Maestripieri et al., 1992; Troisi, 2002), and such activities may help animals cope with frustrating circumstances by allowing them to divert their attention (McFarland, 1966). The results of this study therefore suggest that the number, noise level, and activity level of zoo visitors may be a source of autonomic arousal for this group of primates.

Similarly, we found that the langurs were involved in a greater number of aggressions when visitor impact was higher. This finding was also consistent with our original prediction. Many other species have shown increases the frequency or intensity of intraspecific aggression in the presence of zoo visitors, including lion-tailed macaques (Macaca silenus), golden-bellied mangabeys (Cercocebus galeritus chrysogaster), cotton-topped tamarins (Saguinus oedipus), Diana monkeys (Cercopithecus diana), ring-tailed lemurs (Lemur catta), chimpanzees (Pan troglodytes), Indian blackbucks (Antilope cervicapra), Indian gaurs (Bos gaurus gaurus), and little penguins (Eudyptula minor; Chamove et al., 1988; Findley, 2002; Glatston et al., 1984; Mallapur et al., 2005; Mitchell et al., 1991; Rajagopal et al., 2011; Sekar et al., 2008; Sherwen et al., 2015). Aggression may reflect frustration, and augmented aggression in captive animals is often attributed to stress-inducing environmental conditions (Broom and Johnson, 1993; Chamove et al., 1988; Findley, 2002; Glatston et al., 1984; Rajagopal et al., 2011; Van Loo et al., 2003), an explanation that may also hold true in the current study.

Contrary to our original prediction, we found that the ebony langurs were more likely to exhibit affiliative behaviors (mounting and allogrooming) with greater visitor impact. Many primates including cotton-topped tamarins (Saguinus oedipus), Diana monkeys (Cercopithecus diana), and ringtailed lemurs (Lemur catta) have shown decreases in allogrooming and/or other affiliative behavior types in the presence of zoo visitors (Chamove et al., 1988; Glatston et al., 1984). Given these results from other species, it may seem surprising that the langurs were more likely to exhibit affiliative behaviors when visitor impact was higher. However, mounting and allogrooming may function to reduce physiological or behavioral indices of stress or tension and provide reassurance in primates (Chadwick-Jones, 1989; Dunbar, 2010; Goosen, 1981; Schino et al., 1988), and whitecrowned mangabeys (Cercocebus atys lunulatus) have also been found to exhibit higher frequencies of affiliative behavior types when visitors are present (vs. when they are absent) and when they are louder (Fragata, 2010). Furthermore, visitors appear to have inconsistent effects on allogrooming in 
Fig. 2 Relationships between visitor impact score and whether ebony langurs a exhibited body shaking, $\mathbf{b}$ autogroomed, $\mathbf{c}$ participated in allogrooming (as actor or receiver), and $\mathbf{d}$ participated in mounting (as actor or receiver) during a 5-min focal sample. The logistic regression curves predicted by the models are shown for significant relationships a

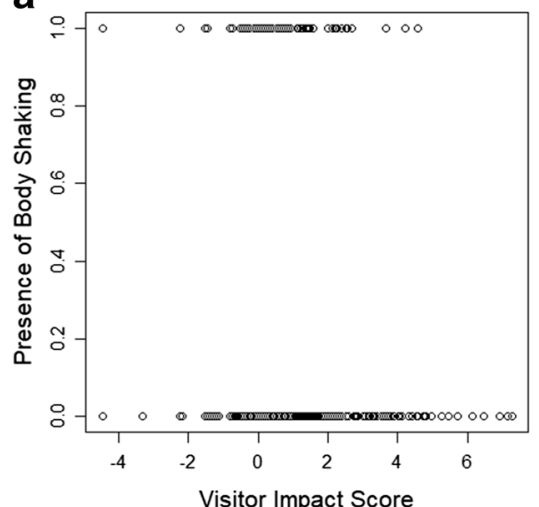

C

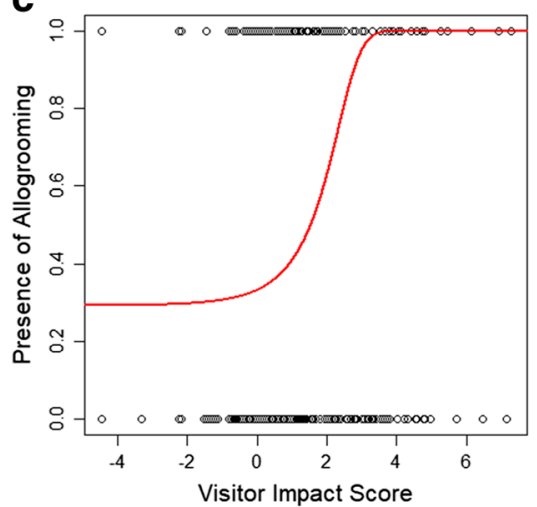

b
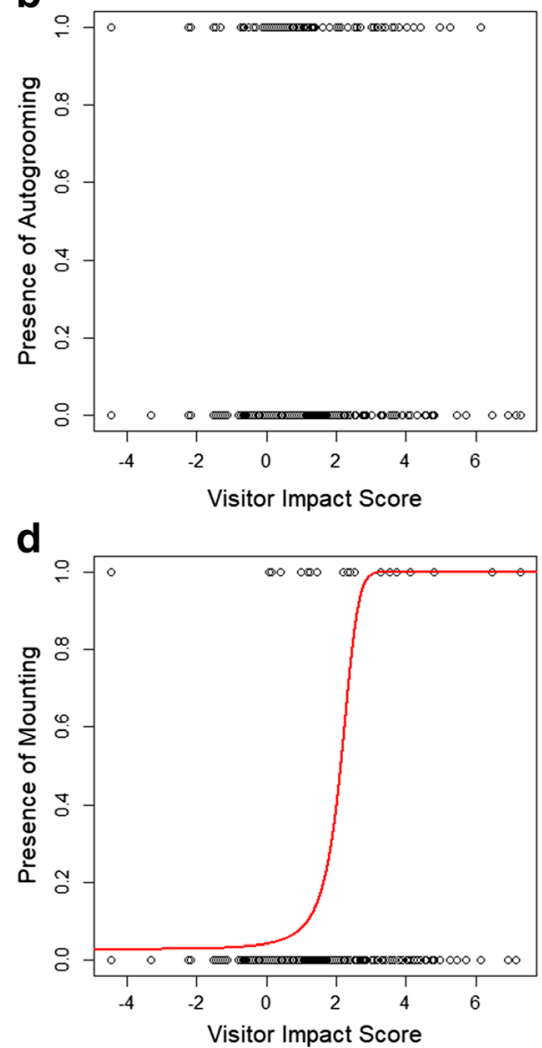

chimpanzees (Pan troglodytes), with one study showing an increase with greater visitor activity (Perret et al., 1995) and another showing a decrease with larger crowds (Wood, 1998). Because affiliative behaviors, like mounting and allogrooming, can reduce the expression of behavioral and

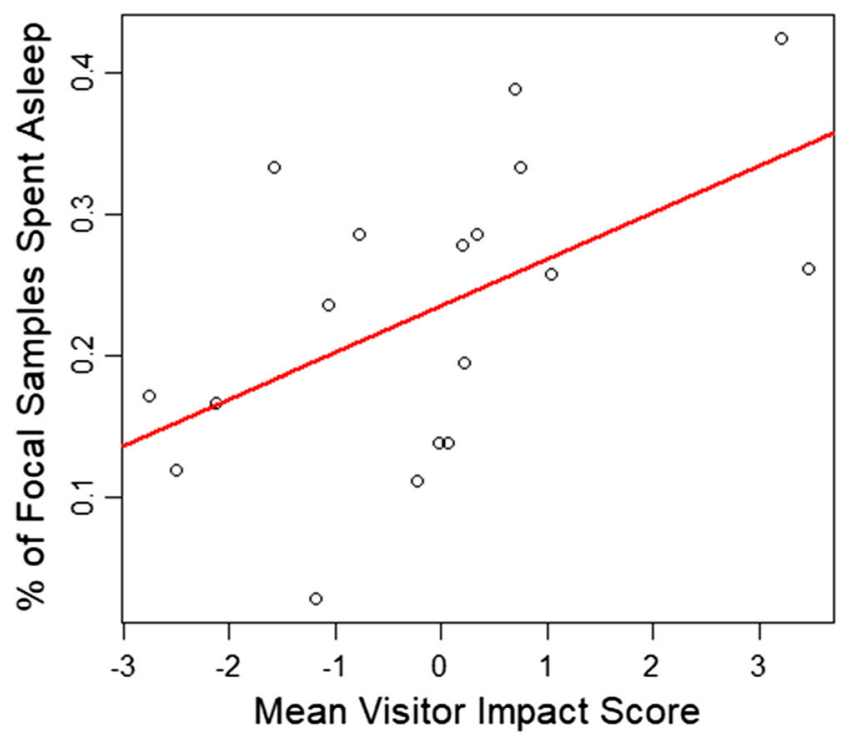

Fig. 3 Relationship between the percentage of focal samples in which the ebony langurs spent sleeping each day and the mean visitor impact score for that day. The regression line predicted by the model is shown physiological indicators of stress in primates (Dunbar, 2010; Goosen, 1981; Schino et al., 1988), it may be the case that the langurs, like other primates, use these activities to alleviate visitor-induced stress (Fragata, 2010).

Although some zoo animals may reduce the amount of time spent resting or sleeping when more visitors are present (Chamove et al., 1988; Rajagopal et al., 2011; Sekar et al., 2008; Todd et al., 2007), the ebony langurs spent more time sleeping on days with higher mean visitor impact scores. This result matched our original prediction. Activities such as sleeping and resting are consistent with learned helplessness - an inability to cope with aversive stimuli that cannot be avoided - and may suggest compromised animal welfare (McGreevy and McLean, 2009; Wells, 2005, 2009; Wells et al., 2002).

Overall, the results from this study suggest that zoo visitors negatively influenced the observed group of captive ebony langurs. These findings match other studies showing that zoo visitors have negative effects on many species of mammals and birds (Chamove et al., 1988; Larsen et al., 2014; Rajagopal et al., 2011; Sekar et al., 2008; Sellinger and Ha, 2005; Sherwen et al., 2015). Although the observed behavioral responses of the langurs likely indicate a stress response, more work is needed to test whether the level of environmental stressors experienced by these monkeys during periods with high visitor impact exceeds natural levels. Moreover, 
we collected data from September to April, thereby missing the busy summer months, which are likely to be accompanied by higher visitor impact scores. If the langurs are more affected by deviations from the typical visitor impact score experienced over several days or weeks than they are by visitor impact score in itself, it is possible that non-summer days with high scores have a stronger effect on behavior than summer days with similarly high visitor impact scores. In other words, these primates may acclimate to higher visitor impact scores during the summer months. Nevertheless, in cases of animal welfare, it may generally be prudent to exercise precaution. Minor changes could be made to the exhibit until future work can shed more light on the situation. It is important to note that, in addition to the overall effect of visitor impact score, visitor number, noise level, and activity level, each affected sleep, displacement activities, and affiliative and aggressive behaviors. Accordingly, any efforts to reduce visitor impact should reduce all three stimuli. The addition of quiet signs combined with frequent sweeps by uniformed personnel could provide a temporary, practical, and cost-effective solution. Past work suggests that the combination of these two measures can significantly reduce the intensity of visitor behavior (Sherwen et al., 2014). Furthermore, limiting the rate of visitors moving through the JungleWorld exhibit would decrease visitor impact.

Lastly, it is critical to consider that the langurs in this study were housed in a mixed species exhibit. The presence of zoo visitors may have an enriching effect on other animal species in the exhibit, and future work should consider this possibility. There is a growing trend towards mixed species exhibits (Pullen, 2010), and in the future, interspecific differences in the visitor effect should be considered during the development of such exhibits.

\section{Conclusion}

This study suggests that zoo visitors had a negative effect on a group of captive ebony langurs. The langurs exhibited higher rates of scratching, displacement yawning, and aggression with higher visitor impact. Additionally, they slept more on days with higher mean visitor impact scores. These results suggest that zoo visitors may be a source of autonomic arousal for the monkeys. However, the langurs were also more likely to participate in mounting and allogrooming when visitor impact was high, indicating that they may employ behavioral mechanisms to decrease visitor-induced stress. Future work should consider if the environmental stress experienced by the ebony langurs under conditions with high visitor impact exceeds natural levels. Until then, it may be wise to minimize visitor impact when this species is on exhibit in zoos.
Acknowledgments We would like to thank the staff at the JungleWorld exhibit at the Bronx Zoo for their cooperation. We would also like to thank Peter McGregor and two anonymous reviewers for their helpful feedback which has led to the improvement of our manuscript.

\section{References}

Broom DM, Johnson KG (1993) Stress and animal welfare. Chapman \& Hall, London

Carder G, Semple S (2008) Visitor effects on anxiety in two captive groups of western lowland gorillas. Appl Anim Behav Sci 115: $211-220$

Chadwick-Jones JK (1989) Presenting and mounting in non-human primates: theoretical developments. J Soc Biol Struct 12:319-333

Chamove AS, Hosey GR, Schaetzel P (1988) Visitors excite primates in zoos. Zoo Biol 74:359-369

Cook S, Hosey GR (1995) Interaction sequences between chimpanzees and human visitors at the zoo. Zoo Biol 14:431-440

Davis N, Schaffner CM, Smith TE (2005) Evidence that zoo visitors influence HPA activity in spider monkeys (Ateles geoffroyii rufiventris). Appl Anim Behav Sci 90:131-141

de Waal FB, Yoshihara D (1983) Reconciliation and redirected affection in rhesus monkeys. Behaviour 85:224-241

Dunbar RI (2010) The social role of touch in humans and primates: behavioural function and neurobiological mechanisms. Neurosci Biobehav Rev 34:260-268

Fernandez EJ, Tamborski MA, Pickens SR, Timberlake W (2009) Animal-visitor interactions in the modern zoo: conflicts and interventions. Appl Anim Behav Sci 120:1-8

Findley ER (2002) The effects of enclosure type on aggressive behavior in captive chimpanzees. Inquiry 3

Fragata M (2010) Visitor behaviours and visitor effects: a case study on the white-crowned mangabey (Cercocebus atys lunulatus) of ZSL London Zoo. [dissertation]. Universidade Nova de Lisboa, Lisbon

Glatston AR, Geilvoet-Soeteman E, Hora-Pecek E, Van Hooff JARAM (1984) The influence of the zoo environment on social behaviour of groups of cotton-topped tamarins, Saguinus oedipus oedipus. Zoo Biol 3:241-253

Goosen C (1981) On the function of allogrooming in Old-World monkeys. In: Chiarelli AB, Corruccini AB (eds) Primate behaviour and sociobiology. Springer Berlin Heidelberg, New York, pp 110-120

Hadidian J (1980) Yawning in an old world monkey, Macaca nigra (Primates: Cercopithecidae). Behaviour 75:133-147

Hosey GR (2000) Zoo animals and their human audiences: what is the visitor effect? Anim Welf 9:343-357

Hosey GR, Druck PL (1987) The influence of zoo visitors on the behaviour of captive primates. Appl Anim Behav Sci 18:19-29

Kutsukake N, Castles DL (2001) Reconciliation and variation in postconflict stress in Japanese macaques (Macaca fuscata fuscata): testing the integrated hypothesis. Anim Cogn 4:259-268

Larsen MJ, Sherwen SL, Rault JL (2014) Number of nearby visitors and noise level affect vigilance in captive koalas. Appl Anim Behav Sci 154:76-82

Maestripieri D, Schino G, Aureli F, Troisi A (1992) A modest proposal: displacement activities as an indicator of emotions in primates. Anim Behav 44:967-979

Mallapur A, Sinha A, Waran N (2005) Influence of visitor presence on the behaviour of captive lion-tailed macaques (Macaca silenus) housed in Indian zoos. Appl Anim Behav Sci 94:341-352

McFarland DJ (1966) On the causal and functional significance of displacement activities. Z Tierpsychol 23:217-235

McGreevy PD, McLean AN (2009) Punishment in horse-training and the concept of ethical equitation. J Vet Behav 4:193-197 
Mitchell G, Herring F, Obradovich S et al (1991) Effects of visitors and cage changes on the behaviours of mangabeys. Zoo Biol 10:417423

Mitchell G, Tromborg CT, Kaufman J et al (1992) More on the 'influence' of zoo visitors on the behaviour of captive primates. Appl Anim Behav Sci 35:189-198

Owen C (2004). Do visitors affect the Asian short-clawed otter in a captive environment. In Proceedings of the 6th Annual Symposium on Zoo Research-BIAZA. p 202-211

Perret K, Preuschoft H, Preuschoft S (1995) Einfluss von Zoobesuchern auf das Verhalten von Schimpansen (Pan troglodytes). Zool Gart 65: 314-322

Pullen K (2010) In the mix. Zooquaria 71:14-15

Quadros S, Goulart VD, Passos L, Vecci MA, Young RJ (2014) Zoo visitor effect on mammal behaviour: does noise matter? Appl Anim Behav Sci 156:78-84

R Core Team (2018) R: a language and environment for statistical computing. R Foundation for Statistical Computing. Austria. URL, Vienna https://www.R-project.org/

Rajagopal T, Archunan G, Sekar M (2011) Impact of zoo visitors on the fecal cortisol levels and behavior of an endangered species: Indian blackbuck (Antelope cervicapra L.). J Appl Anim Welf Sci 14:18-32

Schino G, Scucchi S, Maestripieri D, Turillazzi PG (1988) Allogrooming as a tension-reduction mechanism: a behavioural approach. Am J Primatol 16:43-50

Sekar M, Rajagopal T, Archunan G (2008) Influence of zoo visitor presence on the behavior of captive Indian gaur (Bos gaurus gaurus) in a zoological park. J Appl Anim Welf Sci 11:352-357

Sellinger RL, Ha JC (2005) The effects of visitor density and intensity on the behaviour of two captive jaguars (Panthera onca). J Appl Anim Welf Sci 8:233-244

Sherwen SL, Magrath MJ, Butler KL, Phillips CJ, Hemsworth PH (2014) A multi-enclosure study investigating the behavioural response of meerkats to zoo visitors. Appl Anim Behav Sci 156:70-77
Sherwen SL, Magrath MJ, Butler KL, Hemsworth PH (2015) Little penguins, Eudyptula minor, show increased avoidance, aggression and vigilance in response to zoo visitors. Appl Anim Behav Sci 168:7176

Snyder RL (1975) Behavioural stress in captive animals. In: Research in zoos and aquariums. National Academy of Sciences, Washington D.C, pp 41-76

Todd PA, Macdonald C, Coleman D (2007) Visitor-associated variation in captive Diana monkey (Cercopithecus diana diana) behaviour. Appl Anim Behav Sci 107:162-165

Troisi A (2002) Displacement activities as a behavioural measure of stress in nonhuman primates and human subjects. Stress 5:47-54

Troisi A, Schino G (1987) Environmental and social influences on autogrooming behaviour in a captive group of Java monkeys. Behaviour 100:292-302

Van Loo PLP, Van Zutphen LFM, Baumans V (2003) Male management: coping with aggression problems in male laboratory mice. Lab Anim 37:300-313

Wells DL (2005) A note on the influence of visitors on the behaviour and welfare of zoo-housed gorillas. Appl Anim Behav Sci 93:13-17

Wells DL (2009) Sensory stimulation as environmental enrichment for captive animals: a review. Appl Anim Behav Sci 118:1-11

Wells DL, Graham L, Hepper PG (2002) The influence of length of time in a rescue shelter on the behaviour of kennelled dogs. Anim Welf 11:317-325

Wood W (1998) Interactions among environmental enrichment, viewing crowds, and zoo chimpanzees (Pan troglodytes). Zoo Biol 17:211230

Publisher's note Springer Nature remains neutral with regard to jurisdictional claims in published maps and institutional affiliations. 
Table S1. Effect of visitor number on the frequency of ebony langur aggressions, scratching, and displacement yawning, as well as the occurrence of langur body shaking, autogrooming, allogrooming, and mounting, in focal follows when the focal subject was awake.

\begin{tabular}{lllll}
\hline Behavior & Est. \pm SE & Z & $\mathbf{p}$ & $\mathbf{9 5 \%}$ CI \\
\hline Count response variables & & & & \\
$\quad$ Aggression & $0.775 \pm 0.235$ & 3.302 & $\mathbf{0 . 0 0 1}$ & $0.254-1.194$ \\
Scratching & $0.336 \pm 0.037$ & 9.190 & $<\mathbf{0 . 0 0 1}$ & $0.258-0.414$ \\
$\quad$ Displacement Yawning & $0.399 \pm 0.147$ & 2.711 & $\mathbf{0 . 0 0 7}$ & $0.038-0.674$ \\
Binary response variables & & & & \\
Body Shaking & $0.016 \pm 0.127$ & 0.130 & 0.897 & $-0.232-0.265$ \\
Autogrooming & $0.168 \pm 0.122$ & 1.375 & 0.169 & $-0.082-0.409$ \\
Allogrooming & $0.349 \pm 0.100$ & 3.500 & $<\mathbf{0 . 0 0 1}$ & $0.153-0.545$ \\
Mounting & $0.580 \pm 0.185$ & 3.128 & $\mathbf{0 . 0 0 2}$ & $0.129-0.929$ \\
\hline
\end{tabular}

p-values for significant effects are italicized. See text for details. $(\mathrm{N}=472)$

Table S2. Effect of visitor noise level on the frequency of ebony langur aggressions, scratching, and displacement yawning, as well as the occurrence of langur body shaking, autogrooming, allogrooming, and mounting, in focal follows when the focal subject was awake.

\begin{tabular}{lllll}
\hline Behavior & Est. \pm SE & Z & p & $\mathbf{9 5 \%}$ CI \\
\hline Count response variables & & & & \\
$\quad$ Aggression & $0.720 \pm 0.258$ & 2.792 & $\mathbf{0 . 0 0 5}$ & $0.231-1.570$ \\
Scratching & $0.384 \pm 0.032$ & 11.860 & $<\mathbf{0 . 0 0 1}$ & $0.329-0.456$ \\
Displacement Yawning & $0.577 \pm 0.222$ & 2.602 & $\mathbf{0 . 0 0 9}$ & $0.097-1.137$ \\
Binary response variables & & & & \\
Body Shaking & $0.265 \pm 0.137$ & 1.941 & 0.052 & $-0.003-0.533$ \\
Autogrooming & $0.063 \pm 0.115$ & 0.548 & 0.584 & $-0.165-0.291$ \\
Allogrooming & $0.435 \pm 0.110$ & 3.961 & $<\mathbf{0 . 0 0 1}$ & $0.239-0.670$ \\
Mounting & $1.085 \pm 0.355$ & 3.060 & $\mathbf{0 . 0 0 2}$ & $0.390-1.781$ \\
\hline
\end{tabular}

p-values for significant effects are italicized. See text for details. $(\mathrm{N}=472)$ 
Table S3. Effect of visitor activity level on the frequency of ebony langur aggressions, scratching, and displacement yawning, as well as the occurrence of langur body shaking, autogrooming, allogrooming, and mounting, in focal follows when the focal subject was awake.

\begin{tabular}{lllll}
\hline Behavior & Est. \pm SE & Z & $\mathbf{p}$ & $\mathbf{9 5 \%}$ CI \\
\hline Count response variables & & & & \\
$\quad$ Aggression & $0.674 \pm 0.246$ & 2.735 & $\mathbf{0 . 0 0 6}$ & $0.191-1.156$ \\
Scratching & $0.326 \pm 0.034$ & 9.711 & $<\mathbf{0 . 0 0 1}$ & $0.262-0.403$ \\
Displacement Yawning & $0.658 \pm 0.236$ & 2.791 & $\mathbf{0 . 0 0 5}$ & $0.176-1.175$ \\
Binary response variables & & & & \\
Body Shaking & $0.404 \pm 0.147$ & 2.742 & $\mathbf{0 . 0 0 6}$ & $0.115-0.692$ \\
Autogrooming & $0.044 \pm 0.113$ & 0.389 & 0.697 & $-0.178-0.269$ \\
Allogrooming & $0.402 \pm 0.113$ & 3.553 & $<\mathbf{0 . 0 0 1}$ & $0.198-0.623$ \\
Mounting & $1.045 \pm 0.293$ & 3.571 & $<\mathbf{0 . 0 0 1}$ & $0.453-1.702$ \\
\hline p-values for significant effects are italicized See text for details. $(\mathrm{N}=472)$
\end{tabular}

p-values for significant effects are italicized. See text for details. $(\mathrm{N}=472)$ 